\section{Conhecimentos e práticas de educadoras infantis sobre anemia}

\section{Childcare providers knowledge on anemia}

\section{Abstract}

Objectives: identify and analyze anemia knowledge and practices of daycare center providers.

Methods: study performed in a public daycare center for low income families living in the outskirts of the city of São Paulo. The majority of the children was seen in the health clinic of the daycare center for over six months and came from low income families. All 15 care providers were high school graduates. The interviews were taped, transcribed and submitted to content analysis.

Results: care providers'knowledge and practices stemmed from common sense and day to day experience. Many had wrong concepts with potential harm in terms of care provision. Their perception was that anemia control and prevention depended on "parents education", a perspective that could interfere with the care providers/parents relationship in a sense that the problems are of a social nature and do not depend exclusively on family care. Some identified anemic children and attempted to help but the majority transferred the responsibility to healthcare professionals and did not feel they were related to care giving practices and conditions.

Conclusions: there is a need to educate care providers on anemia and the care of this condition, not only in initial training programs as well as in ongoing training programs.

Key words Anemia, Child day care centers, Child care
Claudia Nery Teixeira-Palombo 1

Elizabeth Fujimori 2

1-2 Departamento de Enfermagem em Saúde Coletiva. Escola de Enfermagem. Universidade de São Paulo. Av. Dr. Enéas de Carvalho Aguiar, 419. São Paulo, SP, Brasil. CEP: 05.403-000

\section{Resumo}

Objetivos: identificar e analisar conhecimentos $e$ práticas de educadoras de uma creche, relativos à anemia.

Métodos: estudo desenvolvido em creche pública que atende população da periferia da cidade de São Paulo. A maioria das crianças freqüentava posto de saúde, creche há mais de seis meses e pertencia a famílias de baixa renda. Todas as 15 educadoras haviam concluído o segundo grau. As entrevistas foram gravadas, transcritas e submetidas à análise de conteúdo.

Resultados: os conhecimentos e práticas das educadoras decorriam do senso comum, sendo adquiridos no dia-a-dia, com a própria experiência. Muitas apresentaram conceitos equivocados, que podem prejudicar a prestação de cuidados. Para elas, o controle e a prevenção da anemia depende da "educação dos pais", perspectiva que pode dificultar a construção da parceria educadores/pais, pois impede a percepção de que os problemas são determinados socialmente e não decorrentes apenas dos cuidados prestados pela família. Algumas reconheciam crianças anêmicas e procuravam contribuir para seu controle, porém a maioria atribuía essa responsabilidade aos profissionais de saúde, não relacionando com as condições e práticas de cuidado de sua competência.

Conclusões: aponta-se a necessidade de incluir conhecimentos sobre anemia e práticas de cuidados, tanto na formação inicial, quanto continuada dos educadores infantis.

Palavras-chave Anemia, Creches, Cuidado da criança 


\section{Introdução}

De acordo com a Organização Mundial da Saúde (OMS), ${ }^{1}$ anemia nutricional é o estado em que a concentração de hemoglobina no sangue é anormalmente baixa em conseqüência da carência de um ou mais nutrientes essenciais, de forma que se consideram anêmicas crianças com níveis de hemoglobina sangüínea inferiores a $11 \mathrm{~g} / \mathrm{dL}$.

A anemia causada pela deficiência de ferro, no entanto, constitui o problema nutricional de maior magnitude na atualidade, não obstante o compromisso e os esforços de organizações internacionais e nacionais para sua redução. Segundo estimativas da World Health Organization (WHO), ${ }^{2}$ nos países não industrializados, quase $50 \%$ das crianças menores de quatro anos são anêmicas, pois representam um dos grupos mais vulneráveis, em decorrência do crescimento acelerado dos tecidos, que impõe maior necessidade orgânica do mineral, aliado ao baixo consumo de ferro, decorrente das precárias condições de vida.

Na cidade de São Paulo, a análise da tendência secular da anemia realizada por Monteiro et al. ${ }^{3}$ revela que a prevalência da anemia entre as crianças com menos de cinco anos aumentou de $22,7 \%$ na década de 70 para $35,6 \%$ na década de 80 e $46,9 \%$ na década de 90 , afetando especialmente as crianças dos estratos socioeconômicos mais baixos da população.

Vale destacar também o estudo de Torres et al.,4 que envolveu 63 municípios do estado São Paulo e constatou que $59,1 \%$ das crianças menores de dois anos, atendidas nos serviços estaduais de saúde, estavam anêmicas, sendo que 25,1\% delas apresentavam anemia grave. Estudos realizados em creches também têm mostrado prevalências elevadas de anemia: $61 \%$ em menores de três anos $(n=271)$ que freqüentavam creches públicas de Cuiabá em período integral, 5 e $81 \%$ em crianças de mesma faixa etária ( $n=324)$, freqüentadoras de creches em Recife. 6

A carência de ferro na criança tem sido associada à diminuição da atividade motora e interação social, sonolência, irritabilidade e incapacidade de fixar a atenção, que podem reduzir a capacidade de aprendizagem e também comprometer o crescimento. ${ }^{7}$

Embora a erradicação completa das deficiências nutricionais dependa da superação dos grandes contrastes econômicos e sociais gerados e mantidos pelo processo de produção e distribuição de bens e serviços, ${ }^{8}$ ações específicas do setor saúde, sobretudo da atenção primária, podem contribuir para melhorar o quadro epidemiológico dessas deficiências. 9
A agenda de compromissos do Ministério da Saúde ${ }^{10}$ para a saúde integral da criança e redução da mortalidade infantil aponta como o primeiro princípio norteador do cuidado na saúde da criança o planejamento e desenvolvimento de ações intersetoriais, incluindo, nesse aspecto, a articulação da unidade de saúde com creches e pré-escolas, cabendo aos serviços de saúde e profissionais engajados na área da saúde coletiva a promoção de parcerias e trabalhos conjuntos com tais equipamentos sociais, na busca da promoção da saúde integral da criança.

Partindo dessa premissa, as creches, que constituem locais em que se presta cuidado integral à criança, representam locais propícios para a efetivação de práticas preventivas.

Tendo em vista essas considerações, o objetivo deste artigo é apresentar os resultados de uma pesquisa sobre conhecimentos e práticas de educadoras de uma creche, relativos à anemia.

\section{Métodos}

Este estudo integrou um projeto maior, de natureza quali-quantitativa, desenvolvido em uma creche municipal conveniada, localizada no distrito de Lajeado, subprefeitura de Guaianases, região mais excluída da zona Leste da cidade de São Paulo, estado de São Paulo, de acordo com o mapa da exclusão social elaborado por Sposati, 11 que demonstra a precariedade das condições socioeconômicas e ambientais da região, marcadas constantemente pelo desemprego, discriminação e violência.

Classificada como de grande porte, a creche tem capacidade para atender 170 crianças menores de cinco anos e funciona de segunda a sexta-feira, das 7 às $17 \mathrm{~h}$, à exceção do mês de janeiro, quando interrompe suas atividades para férias coletivas. $\mathrm{O}$ quadro de funcionários inclui 25 trabalhadores com carga horária de 40 horas semanais, sendo uma diretora, uma coordenadora, uma auxiliar de enfermagem, duas ajudantes de serviços gerais, quatro cozinheiras e 16 educadoras.

Quatro unidades de saúde do Programa de Saúde da Família, que atendem cerca de 4000 famílias cada, localizam-se no entorno da creche, além de uma Escola Municipal de Educação Infantil que atende 1080 crianças de quatro a seis anos, duas outras creches que atendem mais de 150 crianças cada uma e uma escola de ensino fundamental.

Os dados quantitativos relacionados à caracterização das crianças foram colhidos a partir da ficha 
de identificação e entrevistas realizadas com mães/responsáveis pela criança. A coleta dos dados foi feita na própria creche por uma enfermeira e duas alunas de iniciação científica, graduandas de enfermagem, devidamente treinadas em técnicas de entrevista, utilizando-se formulário pré-testado. Os dados pré-codificados foram organizados em bancos do software Excel e analisados utilizando-se o software Epi-info 6.

A Tabela 1 apresenta características gerais das crianças atendidas na creche. Constata-se que a maioria freqüentava a creche há mais de seis meses e pertencia a famílias com renda per capita inferior a 0,5 salário mínimo. Apenas 6,1\% haviam nascido com baixo peso $(<2500 \mathrm{~g})$, mais de $80 \%$ freqüentavam serviços de saúde e haviam sido amamentadas, porém $43,3 \%$ das crianças com exame de sangue recente tinham apresentado anemia.

O cunho qualitativo da pesquisa foi desenvolvido com a preocupação de levantar conhecimentos e práticas das educadoras, relacionados ao aspecto do "vivido", que inclui o universo de crenças, atitudes, motivações e valores, 12 que na área da enfermagem têm contribuído para o aperfeiçoamento da prática profissional.

Embora a composição da amostra, em pesquisa social de abordagem qualitativa, não passe pela representatividade numérica, este estudo envolveu quase a totalidade das educadoras da creche. Participaram do estudo 15 educadoras $(94 \%)$ que foram entrevistadas individualmente utilizando-se roteiro semi-estruturado. O roteiro continha apenas algumas questões-guia para facilitar e aprofundar a comunicação, não cerceando a fala das entrevistadas. Baseou-se em levantar dados sobre conhecimentos e práticas relacionados à anemia, ou seja, o entendimento acerca desse problema, suas causas e conseqüências e as concepções que norteiam a identificação e o cuidado da criança com anemia. As entrevistas tiveram duração média de 30 minutos, e para melhor apreender as informações desejadas, foram gravadas, com autorização das educadoras, transcritas na íntegra e submetidas à análise de conteúdo proposta por Bardin. 13

A faixa etária das educadoras variou de 21 a 29 anos, com média de 28 anos. A maioria morava na própria região e tinha filhos com idade de três meses a nove anos, com média de cinco anos e todas tinham concluído o segundo grau. A maioria das educadoras trabalhava na creche há pelo menos quatro anos e apenas uma delas referiu experiência anterior.

Todos os aspectos ético-legais foram contemplados: o estudo foi aprovado por Comitê de Ética em Pesquisa, autorizado pela direção da creche e a
Tabela 1

\begin{tabular}{|c|c|c|}
\hline Variáveis & $\mathbf{N}$ & $\%$ \\
\hline \multicolumn{3}{|l|}{ Sexo } \\
\hline Masculino & 80 & 54,8 \\
\hline Feminino & 66 & 45,2 \\
\hline \multicolumn{3}{|c|}{ Faixa etária (meses) } \\
\hline$<12$ & 13 & 8,9 \\
\hline $12 \vdash 24$ & 28 & 19,2 \\
\hline $24+36$ & 33 & 22,6 \\
\hline $36+48$ & 44 & 30,1 \\
\hline$\geq 48$ & 28 & 19,2 \\
\hline \multicolumn{3}{|c|}{ Tempo de freqüência à creche (meses)* } \\
\hline$<6$ & 19 & 13,6 \\
\hline$\geq 6$ & 121 & 86,4 \\
\hline \multicolumn{3}{|c|}{ Renda familiar (salário mínimo per capita)* } \\
\hline $0-0,5$ & 89 & 64,0 \\
\hline $0,5-1,0$ & 39 & 28,1 \\
\hline$\geq 1$ & 11 & 7,9 \\
\hline \multicolumn{3}{|c|}{ Peso ao nascer } \\
\hline$<2500 \mathrm{~g}$ & 9 & 6,4 \\
\hline$\geq 2500 \mathrm{~g}$ & 131 & 93,6 \\
\hline \multicolumn{3}{|c|}{ Aleitamento materno* } \\
\hline $\operatorname{Sim}$ & 127 & 88,2 \\
\hline Não & 17 & 11,8 \\
\hline \multicolumn{3}{|c|}{ Freqüência a posto de saúde } \\
\hline Sim & 124 & 84,9 \\
\hline Não & 22 & 15,1 \\
\hline \multicolumn{3}{|c|}{ Palidez palmar } \\
\hline Sim & 10 & 6,8 \\
\hline Não & 136 & 93,2 \\
\hline \multicolumn{3}{|c|}{ Níveis de hemoglobina ( $\mathrm{g} / \mathrm{dL}$ ) de exame recente* } \\
\hline$<9$ & 1 & 3,3 \\
\hline 9 a 10 & 6 & 20,0 \\
\hline 10 a 11 & 6 & 20,0 \\
\hline$\geq 11$ & 17 & 56,7 \\
\hline
\end{tabular}

* Não foram obtidas informações para a totalidade das crianças.

coleta dos dados foi realizada somente após assinatura do Termo de Consentimento Livre e Esclarecido, por pais/responsáveis e educadoras.

\section{Resultados e Discussão}

Sabe-se que o conhecimento sobre o processo saúdedoença associa-se à concepção de mundo, valores, atitudes e crenças da sociedade. ${ }^{12}$ Assim, embora as educadoras estudadas apresentassem algum conhecimento sobre anemia, esse se referia a idéias vagas e fragmentadas, de senso-comum. 
Anemia acho que deve ser alguma coisa no sangue, né?

[...] acho que o sangue num tá bom, tá fraco, né?

É a falta de ferro no sangue, né?

Falta de ferro.

Falta vitamina no sangue.

Entre as anemias nutricionais, a anemia ferropriva, definida como estado em que a concentração de hemoglobina no sangue é anormalmente baixa em consequiência da carência de ferro, é a mais comum. ${ }^{1}$ As educadoras relacionaram anemia a "sangue fraco", algumas até à falta de ferro no sangue. Destaca-se, no entanto, que duas educadoras referiram nada saber sobre esse problema nutricional que afetava cerca de $43 \%$ das crianças da creche.

Quanto às causas, o aparecimento da anemia é atribuído à alimentação inadequada.

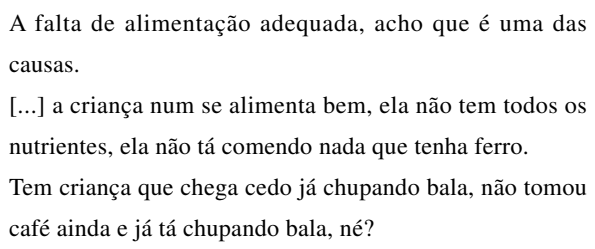

Para as educadoras entrevistadas neste estudo, a compreensão das causas da anemia fica atrelada ao que Monteiro et al. 3 denominam como "determinantes proximais". Esses autores analisaram a tendência secular da anemia na infância na cidade de São Paulo através da adoção de um modelo de determinação, que postula que o risco da anemia depende, em primeira instância, da adequação nutricional do consumo alimentar, isto é, da oferta de ferro, e também do nível de exposição da criança a doenças, particularmente, aquelas associadas à espoliação de ferro, como parasitoses intestinais e malária "determinantes proximais". O consumo alimentar e a exposição a doenças, por sua vez, seriam condicionados por uma série de fatores, que incluem disponibilidade de alimentos, cuidados alimentares e de saúde e saneamento ambiental, ou seja, são "determinantes intermediários". Essas condições dependem fundamentalmente da renda familiar e da capacidade da família alocar racionalmente a renda: são os chamados "determinantes distais".

Martins et al.,8 que buscaram caracterizar a determinação da anemia carencial, a partir de algumas relações biológicas e sociais, mostraram como as condições para a ocorrência da anemia ferropriva estão atreladas às condições sociais e econômicas de classe, seja pelas deficiências qualitativas e quantitativas da dieta, seja pela precariedade do saneamento ambiental, condição típica das áreas habitadas pelas camadas sociais mais baixas. Em outras palavras, a determinação social da anemia pode ser compreendida como resultado de uma espoliação orgânica frente às condições de vida produção e consumo - em que determinada classe social está inserida.

As educadoras não associaram a presença da anemia às condições de vida das crianças. Para a maioria delas, a causa determinante é a alimentação inadequada ou a falta de alimentação, de ferro ou "vitamina no sangue", ou seja, atribuem o desenvolvimento da anemia a um "agente etiológico" específico - falta de ferro/vitaminas -, restringindo a determinação à dimensão biológica, da mesma forma como observou Maranhão, 14 em relação ao processo saúde-doença. Essa compreensão encontra-se atrelada à concepção dominante do processo

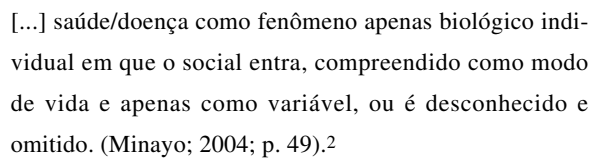

Certamente, o consumo alimentar adequado, a começar com o aleitamento materno exclusivo até os seis meses, e sua manutenção a partir dessa idade até os dois anos, associado a uma alimentação complementar quali e quantitativamente apropriada, contribui para manter os níveis de hemoglobina dentro dos parâmetros de normalidade. 15

Apesar desse conhecimento, uma pesquisa realizada pelo Ministério da Saúde16 revelou que, em 2001, a mediana do aleitamento materno exclusivo nas capitais brasileiras era de apenas 33 dias. No município de São Paulo, o aleitamento materno exclusivo nos primeiros quatro meses raramente alcança índices superiores a 30\%.17 Por outro lado, o leite de vaca na forma líquida ou em pó, que acaba tornando-se a base da alimentação infantil, eleva a ocorrência de anemia por apresentar baixa biodisponibilidade do mineral e provocar microhemorragias intestinais, se consumido no primeiro ano de vida. 18

Assim, cabe aos profissionais de saúde buscar a reversão desse quadro, tanto por meio do trabalho nos serviços de saúde, como também por meio de parcerias com equipamentos sociais, como escolas, pré-escolas, creche, indústrias entre outros grupos da comunidade.

Vale destacar que além das duas educadoras que referiram nada saber sobre anemia, outras duas não responderam esta parte da entrevista por informarem não ter conhecimento sobre causas da anemia. Outras educadoras relacionaram, equivocadamente, 
a anemia a um problema de saúde transmissível, ou seja, que a mãe transmite à criança por meio da lactação, ou até de forma hereditária. Tal concepção certamente interfere de forma negativa sobre a prática do aleitamento materno, não apenas na sua experiência pessoal, porém também no âmbito coletivo, uma vez que as educadoras são responsáveis tanto pelo cuidado quanto pela educação de crianças institucionalizadas e lidam com suas mães/responsáveis.

Olha eu já ouvi falar porque minha menina com um mês deu anemia, porque ela só mamava no peito, daí acho que eu tava com anemia e passou pra ela, né.

Anemia é complicado, porque minha filha sempre foi gordinha e teve anemia, né? Acho que a anemia eu passei pra ela.

Além disso, dois terços das educadoras referiram que anemia pode se transformar em leucemia.

Dizem que pode virar leucemia, né?

A anemia grave dá leucemia [...], né? Então tem que cuidar.

[...] a anemia se não for cuidada vira leucemia, que é um problema muito sério, né?

Com certeza pode até causar leucemia.

A leucemia, classificada como um tipo de anemia causada por falta de produção de hemoglobina, decorre da substituição do tecido hematopoiético normal por um tecido neoplásico, que leva à diminuição da densidade e do número de células hematopoiéticas, ou seja, a leucemia é um outro tipo de anemia, que nada tem a ver com a anemia causada pela deficiência de ferro. ${ }^{19}$

Assim, para educadoras que cuidam diariamente de um coletivo de crianças, cujo principal problema nutricional é a anemia ferropriva, esses são dados extremamente relevantes a serem considerados e demonstram, como destacado por Maranhão, 14 a necessidade da inclusão de conteúdos relacionados à promoção da saúde, tanto na formação dos educadores infantis, quanto na educação continuada em serviço, incluindo aí parcerias com serviços locais de atenção primária, com vistas a aprimorar a qualidade dos cuidado prestado às crianças e reduzir os riscos de adoecimento, tais como infecções respiratórias, maior entre crianças que freqüentam creches. 20

Como o ferro está envolvido na respiração celular, os baixos níveis de hemoglobina interferem no metabolismo energético, podendo afetar o nível de atividade física. No sistema nervoso central o ferro pode estar associado a retardo na capacidade cognitiva da criança. Na pele e nas mucosas, a queda de hemoglobina reflete-se por palidez cutânea, e de mucosas, utilizada para avaliar a presença de anemia em crianças. ${ }^{21}$

Agências internacionais como a Organização Mundial da Saúde e o Fundo das Nações Unidas para a Infância (UNICEF) têm proposto exames baseados em critérios e sinais clínicos simples, como palidez palmar e de conjuntivas para triagem da anemia em crianças de dois meses a cinco anos. ${ }^{22}$

Entretanto, manifestações clínicas como palidez cutânea, cansaço, capacidade de concentração e aprendizado reduzidos, geralmente são discretas nos indivíduos portadores de ferrodeficiência e/ou anemia leve, fato que dificulta a identificação precoce da anemia. 21 Apesar disso, algumas educadoras associaram anemia a alguns desses sinais e sintomas, porém quando solicitadas a identificar alguma criança anêmica, poucas conseguiram cumprir a tarefa. Certamente porque também na creche, a anemia mais prevalente fosse a leve (40\%), de difícil reconhecimento clínico. Das sete crianças apontadas como possivelmente anêmicas, quatro apresentaram exame recente de sangue, que possibilitou comprovar ou não a suspeita das educadoras. Das quatro crianças com exame, nenhuma era anêmica.

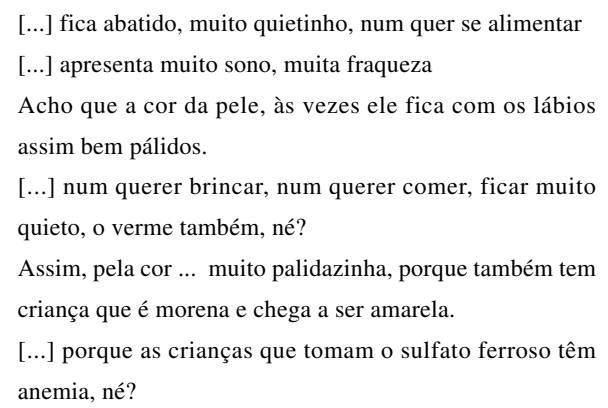

O estudo de Ramos et al.,23 que envolveu 242 crianças menores de dois anos e avaliou a anemia por meio da determinação dos níveis de hemoglobina e palidez palmar como preconizado na estratégia Atenção Integrada às Doenças Prevalentes da Infância (AIDPI), constatou baixa sensibilidade desse sinal clínico na detecção da anemia na amostra estudada, que apresentou elevada prevalência de anemia leve.

Também Spinelli et al.,24 com objetivo de avaliar a confiabilidade e a validade de indicadores clínicos como métodos para triagem de anemia, realizaram estudo em uma creche municipal com 135 crianças de três meses a seis anos de idade. Sinais clínicos de 
palidez palmar e de conjuntivas, observados por uma nutricionista treinada e três funcionárias da creche foram comparados com níveis de hemoglobina obtidos de punção digital. Além de indicarem baixa concordância, os resultados mostraram baixa sensibilidade dos sinais clínicos em relação ao diagnóstico da anemia.

Estudos realizados em várias regiões do país mostram que a anemia leve é a forma mais prevalente em nosso meio. 3,4,15,25 Esse fato justifica a dificuldade em se reconhecer sinais clínicos, que muitas vezes passam desapercebidos pelos próprios profissionais de saúde e pelos pais.

Identificar crianças anêmicas tendo como parâmetro a ingestão de sulfato ferroso, como relatado por uma educadora, também não constitui método seguro, pois, recentemente, a suplementação medicamentosa passou a ser recomendada para todas as crianças a partir dos seis meses. 26

A suplementação de ferro constitui uma das estratégias preconizadas para combater a anemia ferropriva nos grupos populacionais mais susceptíveis. $26 \mathrm{Na}$ cidade de São Paulo, a Secretaria Municipal de Saúde adotou no Programa de Saúde da Família a estratégia da Atenção Integrada às Doenças Prevalentes na Infância como protocolo a ser assumido por médicos e enfermeiros capacitados. 27 Nesse protocolo, o ferro deve ser prescrito a partir do segundo mês de vida para as crianças prematuras e a partir do sexto mês para todas as crianças nascidas a termo, de forma continuada até o $24^{\circ}$ mês de vida, em ambos os casos, na dosagem de $2 \mathrm{mg} / \mathrm{kg}$ de peso corporal por dia.

A eficácia dessa intervenção, no entanto, geralmente é dificultada por dois fatores: os efeitos colaterais da ingestão de ferro medicamentoso por via oral (náuseas, vômito, cólicas abdominais, obstipação ou diarréia) e a inexistência de sinais e/ou sintomas aparentes que estimulem o uso do sulfato ferroso por um período prolongado.

Torres et al., 4 estudando a eficácia da terapêutica com doses profiláticas de sulfato ferroso em 620 crianças de 4 a 36 meses de idade, atendidas em duas unidades de saúde do município de São Paulo, verificaram melhora no estado nutricional de ferro, porém constataram que $47,5 \%$ das crianças não receberam a medicação corretamente, por esquecimento da mãe. Concluíram que apesar de se mostrar eficiente na recuperação dos níveis de hemoglobina, a terapêutica apresenta entraves do ponto de vista operacional, que devem ser superados.

Tais resultados devem ser conhecidos, tanto por parte de mães/responsáveis como também de educadores infantis, de forma que possam contribuir para a prevenção e o controle da anemia, não só estimulando o consumo de alimentos fonte de ferro, como também controlando e acompanhando a ingestão da suplementação medicamentosa do mineral.

O senso comum que permeia o conhecimento das educadoras parece não ser suficiente para um reconhecimento mais preciso da anemia nas crianças, necessário para distinguir uma criança hipoativa de uma criança sonolenta, uma criança pálida de uma criança "branquinha".

Constatou-se que apenas uma educadora havia recebido treinamento específico para trabalhar na creche, ou seja, a grande maioria cuidava das crianças a partir dos conhecimentos e práticas adquiridos com a própria experiência obtida no cotidiano da creche, observando outros educadores trabalhando e também a partir de situações vivenciadas como mães.

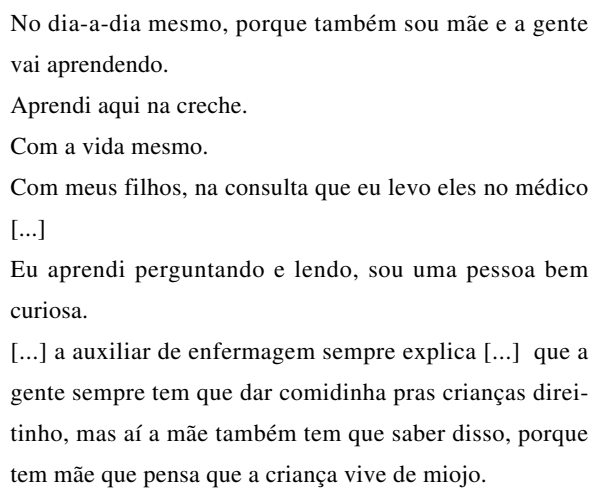

Certamente tais conhecimentos foram assimilados parcialmente e sofreram reinterpretação de acordo com seus conhecimentos prévios, mesmo que fossem idéias vagas, fragmentadas ou ambíguas. ${ }^{12} \mathrm{~A}$ suspeita de anemia era comunicada à auxiliar de enfermagem da creche para que as providências cabíveis fossem tomadas, isto é, comunicação à mãe ou encaminhamento da criança a um serviço de saúde. Apenas algumas educadoras referiram estimular mais a criança nas atividades e observar melhor sua alimentação.

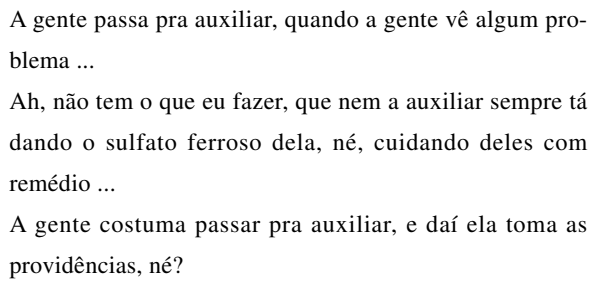

Nessa perspectiva, constata-se que as educadoras não relacionam a promoção da saúde das crianças 
com as condições e práticas de cuidado cotidiano prestados na creche, que são de sua competência.

$\mathrm{Na}$ concepção das educadoras, o problema da anemia pauta-se na "educação dos pais", isto é, poderia ser controlado apenas por meio de informação aos pais sobre a alimentação da criança.

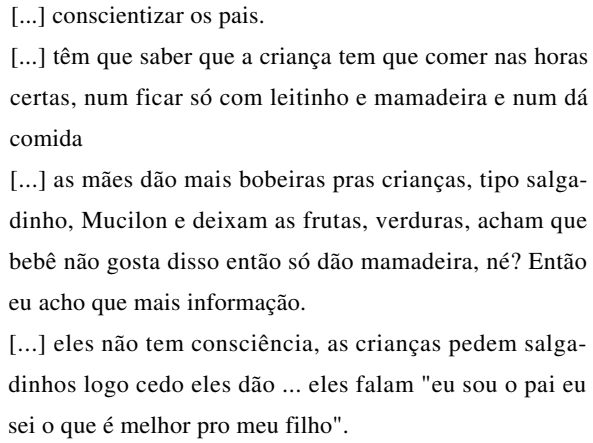

Além dessa percepção sobre os pais, também foram apontadas formas de atuar sobre o problema (palestras, reuniões), acompanhamento de saúde e atuação dos serviços de saúde como medida de combate à anemia.

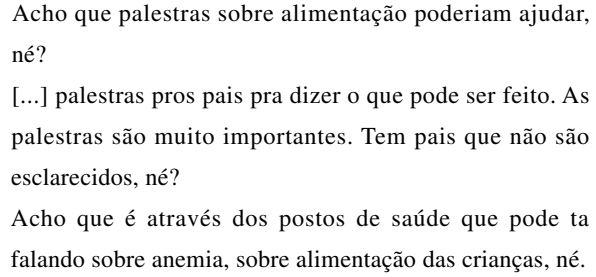

Certamente o aconselhamento nutricional tem impacto positivo, 28,29 porém mais importante que isso é o cuidado prestado pelas educadoras em relação à alimentação da criança na creche. Conforme a faixa etária e a necessidade da criança, supervisionar, auxiliar ou administrar a alimentação, estimulando o consumo de alimentos ricos em ferro e de fatores que favorecem a absorção do mineral, tais como sucos de frutas cítricas, ricas em vitamina C. 30 Nesse sentido, o incremento dos programas de ação continuada e o aprimoramento de ações intersetorias que envolvam articulação entre serviços de saúde e creches locais, devem ser considerados com vistas a prevenir, detectar e controlar essa deficiência nutricional e promover a saúde integral das crianças.

\section{Considerações finais}

Os resultados deste estudo revelaram que as educadoras possuem conhecimentos do senso comum, associados às suas próprias concepções de mundo, costumes, valores e crenças, e sedimentados nas experiências e situações vivenciadas, pois somente uma delas havia recebido treinamento específico para trabalhar na creche. Muitas apresentaram conceitos equivocados em relação à anemia, que podem prejudicar a prestação de cuidados no coletivo.

Para as educadoras, o controle e a prevenção da anemia pautam-se primordialmente pela "educação dos pais", com maiores informações sobre alimentação, via palestra, profissionais e serviços de saúde. Não relacionam com as condições e práticas de cuidado cotidiano prestado na creche, de sua competência. Essa perspectiva de conceber o problema de saúde-doença pode dificultar a construção de uma parceria entre educadores e pais em benefício da criança, uma vez que impede a percepção de que os problemas são determinados socialmente e não somente decorrentes dos cuidados prestados pelas famílias.

O estudo aponta a necessidade de se incluir conhecimentos sobre o processo saúde-doença, especialmente anemia e práticas de cuidados com a saúde das crianças, tanto na formação inicial quanto continuada dos educadores infantis. De uma forma geral, os resultados indicam a necessidade e a importância da parceria e trabalho conjunto entre unidades locais de saúde e creches, com vistas à implementação de ações de promoção à saúde integral da criança, reiterando o primeiro princípio norteador da agenda de compromissos do Ministério da Saúde ${ }^{10}$ para a Saúde Integral da Criança e Redução da Mortalidade Infantil, isto é, planejamento e desenvolvimento de ações intersetoriais.

\section{Agradecimentos}

Às graduandas de enfermagem e bolsistas de iniciação científica do Conselho Nacional de Desenvolvimento Científico e Tecnológico (CNPq), Luciane Simões Duarte e Flavia Antonini Schoeps, pelo apoio na coleta e análise dos dados. 


\section{Referências}

1. OMS (Organización Mundial de la Salud). Anemias nutricionales. Genebra; 1968. (Série de Informes Técnicos, n. 405).

2. WHO (World Health Organization), UNICEF (United Nations International Children's Emergency Fund), UNU (United Nations University). Iron deficiency anaemia assessment, prevention and control: a guide for programme managers. Geneva; 2001.

3. Monteiro CA, Szarfarc SC, Mondini L. Tendência secular da anemia na infância na cidade de São Paulo. Rev Saúde Pública. 2000; 34 (Supl 6): 62-72.

4. Torres AA, Sato K, Queiroz SS. Anemia em crianças menores de dois anos atendidas nas unidades básicas de saúde no Estado de São Paulo, Brasil. Rev Saúde Pública. 1994; 28: 290-4.

5. Brunken GS, Guimarães LV, Fisberg M. Anemia em menores de três anos que freqüentam creches públicas em período integral. J Pediatr. (Rio J) 2002; 78: 50-6.

6. Perez JLP, Gonçalves BPB, Figueiroa FV, Barreto LL, Medeiros JJA, Perez EP, Rocha JA, Brasileiro MC, Silva MMM, Moreira Z. Anemia em crianças menores de três anos - estudo em creches do Recife, PE. Rev IMIP. 1998; 12: $19-24$.

7. Walter T. Conseqüências não hematológicas da deficiência de ferro. Anais Nestlé. 1996; 52: 25-35.

8. Martins IS, Alvarenga AT, Siqueira AAF, Szarfarc SC, Lima FD. As determinações biológica e social da doença: um estudo de anemia ferropriva. Rev Saúde Publica. 1987; 21 : 73-89.

9. Batista Filho M, Rissin A. Deficiências nutricionais: ações específicas do setor saúde para o seu controle. Cad Saúde Pública. 1993; 9: 130-5.

10. Ministério da Saúde. Agenda de compromissos para a saúde integral da criança e redução da mortalidade infantil. Brasília (DF); 2004.

11. Sposati A, organizador. Mapa da exclusão/inclusão social da cidade de São Paulo. São Paulo: Educ; 1996.

12. Minayo MCS. O desafio do conhecimento: pesquisa qualitativa em saúde. 8. ed. São Paulo: Hucitec; 2004.

13. Bardin L. Análise de conteúdo. Lisboa: Edições 70; 1979.

14. Maranhão DG. O processo saúde-doença e os cuidados com a saúde na perspectiva dos educadores infantis. Cad Saúde Pública. 2000; 16: 1143-8.

15. Assis AMO, Barreto ML, Gomes GSS, Prado MS, Santos NS, Santos LMP, Sampaio LR, Ribeiro RC, Oliveira LPM, Oliveira VA. Chidhood anemia prevalence and associated factors in Salvador, Bahia, Brazil. Cad Saúde Pública. 2004; 20: 1633-41.

16. Ministério da Saúde. Secretária de Políticas de Saúde. Área de Saúde da Criança. Prevalência do aleitamento materno nas capitais brasileiras e no Distrito Federal. Brasília (DF);
2001.

17. Venâncio SI, Escuder MML, Kitoto P, Rea MF, Monteiro CA. Freqüência e determinantes do aleitamento materno em municípios do Estado de São Paulo. Rev Saúde Pública. 2002; 36: 313-8.

18. Levy-Costa RB, Monteiro CA. Consumo de leite de vaca e anemia na infância no município de São Paulo. Rev Saúde Pública. 2004; 38: 797-803.

19. Barreto OCO. Como diagnosticar e tratar anemias. Rev Bras Med. 1992; 49: 711-33.

20. Barros AJD. Child-care attendance and common morbidity: evidence of association in the literature and questions of design. Rev Saúde Pública. 1999; 33: 98-106.

21. DeMaeyer EM, Dallman P, Gurney JM, Hallberg L, Sood SK, Srikantia SG. Preventing and controlling iron deficiency anaemia through primary health care. Geneva: WHO; 1989.

22. Ministério da Saúde. Atenção Integrada às Doenças Prevalentes na Infância. Avaliar e classificar a criança doente de dois meses a cinco anos de idade: curso de capacitação. Brasília (DF); 1999.

23. Ramos CA, Fujimori E, Montero RMJM, Minagawa AT, Laurenti D, Oliveira IMV. Utilidade da palidez palmar na detecção da anemia em crianças menores de dois anos. Acta Paul Enferm. 2004; 17: 38-44.

24. Spinelli MGN, Souza JMP, Souza SB, Sesoko EH. Confiabilidade e validade da palidez palmar e das conjuntivas como triagem da anemia. Rev Saúde Pública. 2003; 37: 404-8.

25. Osório MM, Lira PIC, Batista-Filho M, Ashworth A. Prevalence of anemia in children 6-59 months old in the state of Pernambuco, Brazil. Rev Panam Salud Pública. 2001; 10: 101-7.

26. Ministério da Saúde. Programa Nacional de Suplementação de Ferro. Portaria no 730/GM, 13 de maio de 2005. Disponível em http://dtr2001.saude.gov.br/sas/ PORTARIAS/Port2005/GM/GM-730.htm [2005 ago 1].

27. São Paulo (Capital). Secretaria de Saúde. Portaria 3522/2002/SMS. Rotinas e condutas que integram a estratégia Atenção Integrada às Doenças Prevalentes na Infância - AIDPI. Diário Oficial Município [São Paulo] DOM. 2002 Sep 18: 37.

28. Santos I, Victora CG, Martines J, Gonçalves H, Gigante DP, Valle NJ, Pelto G. Nutrition counseling increases weight gain among Brazilian children. J Nutr. 2001; 131: 2866-73.

29. Valle NJ, Santos IS, Gigante DP. Intervenções nutricionais e crescimento infantil em crianças de até dois anos de idade: uma revisão sistemática. Cad Saúde Pública. 2004; 20: $1458-67$.

30. Giugliani ERJ, Victora CG. Normas alimentares para crianças brasileiras menores de dois anos: bases científicas. Brasília (DF): OPS; 1997.

Recebido em 5 de agosto de 2005.

Versão final apresentada em 22 de maio de 2006

Aprovado em 29 de maio de 2006 